\title{
Contribution to lichen flora of Ukrainian Carpathians
}

\author{
COPPINS BRAIAN JOHN \\ KONDRATYUK SERGIY YAKOVYCH \\ KHODOSOVTSEV ALEXANDER YEVGENOVICH \\ ZELENKO SERGEY DMITROVICH \\ WOLSELEY PATRICIA ANNE
}

Coppins B.J., Kondratyuk S.YA., Khodosovtsev A.Ye., Zelenko S.D., Wolseley P.A.. 2005: Contribution to lichen flora of Ukrainian Carpathians. Chornomor. Botan. Journ., vol. 1, № 2: 05-23.

Data on 55 lichen species (namely Absconditella lignicola Vězda \& Pišut, Agonimia tristicula (Nyl.) Zahlbr., Anisomeridium polypori (Ellix et Everh.) M.E. Barr, Arthopyrenia salicis A. Massal., Arthonia ilicina Taylor, A. vinosa Leight., Bacidia circumspecta (Nyl. ex Vainio) Malme, Biatora chrysantha (Zahlbr.) Printzen, B. epixanthoides (Nyl.) Diederich, Catillaria alba Coppins \& Vězda, C. erysiboides (Nyl.) Th. Fr., Cladonia norvegica Tønsberg \& Holien, Diploschistes gypsaceus (Ach.) Zahlbr., Eopyrenula avellanae Coppins, Fellhanera subtilis (Vězda) Diederich \& Sérusiaux, Fuscidea arboricola Coppins \& Tønsberg, F. cyatoides (Ach.) V. Wirth \& Vězda var. corticola (Fr.) Kalb, F. pusilla Tønsberg, Ropalospora viridis (Tønsberg) Tønsberg, Jamesiella anastomosans (P. James \& Vězda) Lücking, Sérusiaux \& Vězda, Hypocenomice caradocensis (Leighton ex Nyl.) P. James \& G. Schned., H. xanthococca (Sommerf.) P. James \& G. Schneider, Lecania cyrtellina (Ach.) Th. Fr., Lecanora cinereofusca Magnusson, L. farinaria Borrer in Hook., Lecidea swartzioidea Nyl., Lepraria lobificans Nyl., L. rigidula (B. de Lesd.) Tønsberg, Leproloma vouauxii (Hue) J.R. Laundon, Leptogium teretiusculum (Wallr.) J.R. Laundon, Leptorhaphis maggiana (A. Massal.) Korber, Megalaria pulverea (Borrer) Hafellner \& E. Schreiner, Micarea adnata Coppins, M. hedlundii Coppins, M. melaeniza Hedl., M. nigella Coppins, M. peliocarpa (Anzi) Coppins \& R. Sant., Mycoblastus sterilis Coppins \& P. James, Microcalicium ahlneri Tibell, Ochrolechia szatalensis Verseghy, Pertusaria pupillaris (Nyl.) Th. Fr., Phaeophyscia endophoenicea (Harm.) Moberg, Ph. hirsuta (Mereschk.) Moberg, Physcia vitii Nadv., Porina leptalea (Durieu \& Mont.) A.L. Sm., Ramonia chrisophaea (Pers.) Vězda, Reichlingia leopoldii Diederich \& Scheidegger, Rinodina griseosoralifera Coppins, $R$. efflorescens Malme, Telocarpon strasseri Zahlbr., Shismatomma ricasolii (A. Massal.) Egea, Trapelia corticola Coppins \& P. James, Trapeliopsis pseudogranulosa Coppins \& P. James, Vezdaea aestivalis (Ohl.) Tsch.-Woess \& Poelt, Zamenhofia hibernica (P. James \& Swinscow) Clauz. \& Roux) found in the territory of the recently created Uzhansky National Nature Park, Ukrainian part of the 'Eastern Carpathian' Trilateral Biosphere Reserve, which for the first time recorded for the whole Ukraine or Eastern Carpathians, are provided. Each taxon is provided by references on papers where full diagnosis is published, list of localities, taxonomical remarks and data on general distribution.

Keywords: lichens, distribution, Ukrainian Carpathians

Ключові слова: лишайники, поширення, Украӥнські Карпати

\section{Introduction}

Within three-year special study of the lichen-forming, lichenicolous and allied fungi of the recently created Uzhansky National Nature Park (N.N.P.), Ukrainian part of the 'Eastern Carpathian' Trilateral Biosphere Reserve (TBR), 503 species belonging to 145 genera and 61 families are found. Totally more than 300 lichen species were for the first time recorded for the territory of the Uzhansky N.N.P. [КОНДРАТЮК та ін. 1998; COPPINS et al. 1998; KONDRATYUK et al., 1998; KONDRATYUK \& COPPINS 1999 a, b; KONDRATYUK, COPPINS, 2000]. Totally about $18 \%$ of them, i.e. 93 species are for the first time recorded for the whole 
Ukraine. The aim of this paper is to provide data on 55 lichen species newly recorded for the whole or Eastern Carpathians (see also KONDRATYUK et al. 2003) Ukraine. Each taxon is provided by references on papers where full diagnosis is published, list of localities, taxonomical remarks and data on general distribution.

The peculiarity of the situation with Uzhansky N.N.P. is connected with that it is one of the best studied in lichenological respect protected territory of the Eastern Carpathians while it is one of the most recent formation among territories mentioned of this region (created only in 1999). In this connection the Uzhansky N.N.P. has favourable conditions for organizing of monitoring on its territory [KONDRATYUK, COPPINS, 2000].

\section{Survey area}

Uzhansky National Nature Park (NNP) is situated in the Eastern Beskydy Mts. on the border of Ukraine with Poland and Slovakia. It was created with its present borders in 1999. The park covers the right-bank part of the Uzh river basin, from village Zabrod' to Uzhok pass. On the west the park border lies as the frontier with Slovakia $(28 \mathrm{~km})$ passes. on the north - as the frontier with Poland $(30 \mathrm{~km})$. The park area lies between $208 \mathrm{~m}$ and $1304 \mathrm{~m}$ a.s.l. [STOYKO et al., 1998]. The geological basis of the park area is made up + of the Upper Cretaceous and Palaeogenic Flysch deposits. The relief of mountains ridges was formed mainly by denudation of local geological structures. Its most striking feature is steep-sided slopes. Within the borders of the park four denudational levels can be considered namely: lower riverside, upper riverside, subpolonyny and polonyny. On the polonyny denudation level the highest summits lie - Kremenetz' (1221 m), Ravka (1304 m), Stinka (1212 m). The park area lies within two thermal zones: moderate humid $(350-800 \mathrm{~m})$ where the sum of active temperatures is higher than 10 is $1800-2000^{\circ} \mathrm{C}$; fresh humid $(800-1300 \mathrm{~m})$, where such amount is equal to $1400-1800^{\circ} \mathrm{C}$ [АНДРИАНОВ, 1968]. Uzhansky NNP lies in the drainage area of the Uzh river. Vegetation of the park is typical for the Beskydy Mts., within altitudes 350-1100 m a broad vegetational belt of beech forests prevail - Fagetum sylvaticae, AceretoFagetum, Sorbeto-Aceretum. Above the belts of the beech forests and green alder shrubs till the highest tops the vegetations belt of subalpine meadows is spreaded [STOYKO et al., 1998].

\section{Materials and Methods}

Seven Darwin Expeditions to the Uzhansky NNP were carried out in June, JulyAugust, and September-October 1997 as well as May-June, July-August and September 1998 respectively. The lichen-forming, lichenicolous fungi and allied fungi species associated with the Red Data Book lichen species Lobaria pulmonaria (L.) Hoffm. were collected and registered during excursions to the territory of Novostuzhytsia, Zornava, Stavne and Kostryno forestris, and the Velyky Berezny collective farm forestry. The specimens of a reported species kept in E and KW.

Abbreviations to the collectors are the following: BJC - B.J. Coppins, CA - A. Coppins, CS - C. Scheidegger, JH - J. Hermannson, IK - I. Kaprusj, KA - A.Ye. Khodosovtsev, KNS - N.S. Kondratyuk, KS - S.Ya. Kondratyuk, LAA - A.A. Levanetz, MOG - O.G. Maryskevich, PAW - P.A. Wolseley, RAA - O.O. Redchenko, TLO - L.O. Tasenkevich, ZSD - Zelenko S.D.

\section{Absconditella lignicola Vězda \& Pišut}

\section{List of taxa}

Description: VĚZDA, PIŠUT, 1984.

Distribution in Ukraine: Zakarpatska oblast, Velykoberezny district, S of Kostrino village, 'Yasynny' ridge, a. $1000 \mathrm{~m}$ alt., 26.09.1997, KS, KA, ZSD (9834) (KW).

Distinguished from Dimerella pinetii by the absence of Trentepohlia as photobiont, etc. 
General distribution: Europe (from British Isles, France, and Portugal to Estonia, Lithuania, Slovakia) and North America.

\section{Agonimia tristicula (Nyl.) Zahlbr.}

Description: PURVIS et al., 1992.

Distribution in Ukraine: Zakarpatska oblast, Velykoberezny district, Novostuzhytzia forestry (the former Stavne forestry) distr. 5, area 3, Mt Cheremkha, a. $1060 \mathrm{~m}$ alt., $49^{\circ} 02^{\prime} \mathrm{N}$, $22^{\circ} 41^{\prime} \mathrm{E}$, old beech forest at NE slope to village Lyuta side with Lobaria, on bark of Fagus sylvatyca, 4.08.1997, KS, BJC et al., (SW of tag 2056) (E); Novostuzhytzia forestry, ridge unnamed between 'Sukha Potochyna' and 'Husariv' Sreams, distr. 15 (13), over mosses on old Fagus sylvatyca, 2.10.1997, KS, KA, ZSD, (tag 2106) (KW); Velykoberezny collective farm forestry, Mt Stinka, $1057 \mathrm{~m}$ alt., $49^{\circ} 00^{\prime} \mathrm{N}, 22^{\circ} 31^{\prime} \mathrm{E}$, near the border with Slovakia, on sandstone, 5.08.1997, ВJC, $A C, K A$ (loc. 9757) (KW). AR Crimea [ХодосовцЕВ, 2002a,б, 2003; ХОДОСОВЦЕВ, РЕДЧЕНКО, 2002].

This species distinguished from Agonimia allobata by minutely squamulose thallus $(0,1-1,0 \times 0,1-0,3(-0,5) \mathrm{mm}$ across $), 1-2$ spores per ascus and their much larger size (60-) 80 $-120(-150) \mu \mathrm{m}$. All specimens studied were sterile.

General distribution: This lichen is widespread in central, western and southern Europe, and is also known from Macaronesia, North America and the Philippines; it also occurs in the Alps, and has a mainly western distribution in Europe [DEGELIUS, 1992; NIMIS, 1993].

\section{Anisomeridium polypori (Ellix et Everh.) M.E. Barr}

Syn. Anisomeridium nyssaegenum (Ellis \& Everh.) R.G. Harris

Description: PURVIS et al., 1992.

Distribution in Ukraine: Zakarpatska oblast, Velykoberezny district, Kostrino forestry, distr. 21, area 2, botanical reserve, 'German' Stream, at the bottom of valley, on bark of Corylus avellanae, $48^{\circ} 55^{\prime} \mathrm{N}, 22^{\circ} 34^{\prime} \mathrm{E}$, alt. $350 \mathrm{~m}, K S, B J C, 29.07 .1997$ (loc. 9742) (E, KW); areas 20 and 23, 11.07.1998 KS (tag 2249); Bystrytske forestry, between villages Chornoholovka and Lyuta, 7-8 km NE from village Chornoholovka, valley of Lyutyanka River, distr. 22, a. $350 \mathrm{~m}$ alt., on bark of Acer platanoides, 6.06.1998, KS, BJC, PAW, ZSD, $R A A, L A A(\mathrm{KW})(\operatorname{tag} 2191)$; distr. 19, a. $290 \mathrm{~m}$ alt., on bark of Fraxinus, 6.06.1998, KS, BJC, $P A W, Z S D, R A A, L A A$ (tag 2195) (KW); Lyutyanske forestry, SW of Lyuta village, distr. 15, 'Shyroky' Stream, on bark of Acer pseudoplatanus, 5.06.1998, KS, BJC, PAW, ZSD, RAA, $L A A(\operatorname{tag} 2182)(\mathrm{KW})$.

The species of the genus Anisomeridium related to Arthopyrenia, but first genus has cellular perithecial wall which not containing bark cells, ascospores without perispore, etc. Some specimens of the genus Anisomeridium are in need of the further studying because they were found with pycnidia only.

General distribution: It is known in Europe (Norway, England, Denmark, Austria, Lithuania) Macaronesia, North Ameria Asia (Japan), and Australia.

\section{Arthonia ilicina Taylor}

Description: PURVIS et al., 1992.

Distribution in Ukraine: Zakarpatska oblast, Velykoberezny district, Novostuzhytzia forestry, at the bottom of the valley, 'Semeniv'-'Sokoliv' Stream, $49^{\circ} 03^{\prime} \mathrm{N}, 22^{\circ} 35^{\prime} \mathrm{E}$, a. $470 \mathrm{~m}$ alt., on bark of Fraxinus, 30.07.1997, KA, CA, ZSD (E).

This species is characterized by (4-)5-6(-7) - septate spores, 26-36 x 10-13 $\mu \mathrm{m}$, concolorous hypothecium and red-brown epithecium $(\mathrm{K}+$ greenish). Tasmania.

General distribution: Europe, North America, Macaronesia, South Africa and 


\section{Arthonia vinosa Leight.}

Description: PURVIS et al., 1992.

Distribution in Ukraine: Zakarpatska oblast, Velykoberezny district, Lyutyanske forestry, 'Rakivska yama', SW of Lyuta village, distr. 20, $1050 \mathrm{~m}$ alt., on bark of Acer pceudoplatanus, 4.06.1998, KS, BJC, ZSD, RAA, LAA (tag 2171, loc. 9841); to $\mathrm{E}$ of 'Rakivska yama', SW of Lyuta village, distr. 20, 1050-1150 m alt., N, NNE slopes, beechsycamore forest, on bark of Fagus, 4.06.1998, KS (loc. 9843); Lyutyanske forestry, SW of Lyuta village, distr. 18, 'Mashyn' Stream, 615-620 m alt., on bark of Fagus, 5.06.1998, KS, $B J C, P A W, Z S D, R A A, L A A$ (tag 2178).

From Arthonia didyma with fleck-like apothecia and growing mainly on smooth bark this species differs in the convex apothecia up to $140 \mu \mathrm{m}$ tall and habitat on rough bark.

General distribution: Europe, Asia (Japan), North America and New Zealand.

\section{Arthopyrenia salicis A. Massal.}

Description: PURVIS et al., 1992.

Distribution in Ukraine: Zakarpatska oblast, Velykoberezny district, Kostrino forestry, distr. 21, area 2, botanical reserve, 'German' Stream, at the bottom of valley, on Fagus branch, $48^{\circ} 55^{\prime} \mathrm{N}, 22^{\circ} 34^{\prime} \mathrm{E}$, alt. $350 \mathrm{~m}, K S, B J C$ et al., 29.07.1997, (loc. 9742) (E, KW); Novostuzhytzia forestry (the former Stavne forestry) distr. 5, area 3, Mt Cheremkha, a. 1060 $\mathrm{m}$ alt., $49^{\circ} 02^{\prime} \mathrm{N}, 22^{\circ} 41^{\prime} \mathrm{E}$, old beech forest at NE slope to village Lyuta side with Lobaria, on bark of Fagus branch, 4.08.1997, KS, BJC et al. (E).

The facultatively lichenized species related with Naetrocymbe punctiformis but differ by lacking pseudoparaphyses and possessing unbranched periphysoids 7-15 x 1-1,5 $\mu \mathrm{m}$.

General distribution: Europe (British Isles, France, Iberian Peninsula and Balearic Islands) and North America.

\section{Bacidia circumspecta (Nyl. ex Vainio) Malme}

Description: PURVIS et al., 1992.

Distribution in Ukraine: Zakarpatska oblast, Velykoberezny district, Novostuzhytzia forestry, ridge 'Yasynny', distr. 4 (20), on bark of Fagus, 26.09.1997, KS, KA, ZSD (KW); Velyky Berezny collective farm forestry, Mt Stinka, at the bottom of Mt, on bark of Fagus, 5.08.1997, BJC, CA, KA (loc. 9757).

It differs from Bacidia subincomta by much shorter ascospores $20-30 \times 2-2,5 \mu \mathrm{m}$, pale coloured to colourless hypothecium and from other species by $\mathrm{K}+$ green epithecium and nonacicular short ascospores.

General distribution: It is rarely collected species: Europe (Great Bretain, Austria), Asia (Turkey), North Africa (Morocco), and North America.

\section{Biatora chrysantha (Zahlbr.) Printzen}

Syn.: Lecidea gyrophorica Tønsberg

Description: TøNSBERG, 1992; PRINTZEN, 1995.

Distribution in Ukraine: Zakarpatska oblast, Velykoberezny district, Novostuzhytzia forestry (the former Stavne forestry) distr. 5, area 3, Mt Cheremkha, alt. $1060 \mathrm{~m}$ alt., $49^{\circ} 02^{\prime} \mathrm{N}$, $22^{\circ} 41^{\prime} \mathrm{E}$, old beech forest at NE slope to Lyuta village with Lobaria, on Fagus, 4.08.1997, KS, BJC et al., (tag 2039) (E, KW); Novostuzhytzia forestry, ridge unnamed between 'Sukha Potochyna' and 'Husariv' Sreams, distr. 15 (13), on Fagus, 27.09.1997, BJC, KS et al. (tags 2066, 2072) (KW); on Fagus, 2.10.1997, KS, KA, ZSD (tags 2105, 2113) (KW); distr. 14 (15), 1000 m alt., on Fagus, 30.05.1998, KS, BJC, PAW, CS, JH, KA, ZSD et al. (tag 2150; loc. 9833); Novostuzhytzia forestry, distr. 2 area 10, near Mt Semenova, sycamore-beech forest with Lobaria, on Acer pseudoplatanus, 28.07.1997, KS et al. (loc. 9741, tag 2008); Lyutyanske forestry, 'Rakivska yama', SW of Lyuta village, distr. 20, $1050 \mathrm{~m}$ alt., on Acer pseudoplatanus, 4.06.1998, KS, BJC, ZSD, RAA, LAA (tag 2171; loc. 9841). 
The specimens collected were sterile. The pale greenish soralia with $\mathrm{C}+$ red reaction distinguish this species from the other (see note to Bacidia epixantoides).

General distribution: This species is hitherto known from northern and northwestern Europe and North America.

\section{Biatora epixanthoides (Nyl.) Diederich}

Description: PURVIS et al., 1992.

Distribution in Ukraine: Zakarpatska oblast, Velykoberezny district, Novostuzhytzia forestry (the former Stavne forestry) distr. 5, area 3, Mt Cheremkha, a. $1060 \mathrm{~m}$ alt., $49^{\circ} 02^{\prime} \mathrm{N}$, $22^{\circ} 41^{\prime} \mathrm{E}$, old beech forest at NE slope to village Lyuta with Lobaria, on Fagus, 4.08.1997, KS, BJC et al. (tag 2047) (E); Novostuzhytzia forestry, ridge unnamed between 'Sukha Potochyna' and 'Husariv' Sreams, distr. 15 (13), on old stamp of Fagus, 2.10.1997, KS, KA, ZSD (KW); Novostuzhytzia forestry, ridge unnamed between 'Sukha Potochyna' and 'Husariv' Streams, distr. 14 (15), on Acer platanoides, 6.06.1998, KS, BJC, PAW, ZSD, RAA, $L A A$ (9833; tag 2152); Lyutyanske forestry, SW of Lyuta village, distr. 18, 'Mashyn' Stream, 615-620 m alt., on Acer psevoplatanus, 5.06.1998, KS, BJC, PAW, ZSD, RAA, LAA (loc. 9844); Bystrytske forestry, between villages Chornoholovka and Lyuta, 3-5 km NE from village Chornoholovka, valley of Lyutyanka River, distr. 19, a. $290 \mathrm{~m}$ alt., on Acer pseudoplatanus, 6.06.1998, KS, BJC, PAW, ZSD, RAA, LAA (9846; 2187).

Fertile specimens of this sorediate species were collected during our study. The greygreen thallus with effuse, often confluent, pale buff soralia with negative test on chemical examination are main characters of this species. Morphologically similar species Lecidea efflorescens has soralia $\mathrm{Pd}+$ red reaction and Biatora chrysantha has soralia $\mathrm{C}+$ red.

General distribution: Europe (Luxembourg, Slovakia) and North America.

\section{Catillaria alba Coppins \& Vězda}

Description: VĚZDA, 1993.

Distribution in Ukraine: Zakarpatska oblast, Velykoberezny district, between willages Stavne an Lubnya, Stavne forestry, distr. 26, area 1, in the upper part of ridge near Lyskovets Stream, on Abies, 3.08.97, BJC, KS, ZSD \& PAW (loc. 9749) (KW, E); Novostuzhytzia forestry, distr. 17, area 19, 'Senychiv' Stream, in the upper part of mountain, old beech forest with Lobaria near areas 11 and 15, on Abies, 24.06.1997, KS (KW) (loc. 9729).

It is very characteristic species with thin grey green continuous thallus in contrast white small dispersed apothecia to $0,1 \mathrm{~mm}$ diam. in cracks of Abies.

General distribution: It was recently described species and hitherto known from Great Britain, Germany, Austria, Italy, Czech Republic, Poland and Slovakia.

\section{Catillaria erysiboides (Nyl.) Th. Fr.}

Description: COPPINS, 1983.

Distribution in Ukraine: Zakarpatska oblast, Velykoberezny district, Volosyanske forestry, 'Adamiv Forest', distr. 22 (area 1), SW of Tykhy village, plantation of Abies, 600$850 \mathrm{~m}$ alt., on Abies, 2.06.1998, KS, BJC, PAW, JH, RAA, LAA (loc. 9836) (E); S of Kostrino village, 'Yasynny' ridge, a. $1000 \mathrm{~m}$ alt., 26.09.1997, KS, KA, ZSD (9834) (KW).

It is very similar to Micarea prasina, but Catillaria erysiboides has non-micareoid photobiont. It has small, plane to convex, reddish brown apothecia that are marginate when young, the exipulum is composed of much-branched, radiating hyphae which are distinct in $\mathrm{K}$ but still tightly bound by the gel matrix, and the spores are ovoid and often constricted at the septum, 1-septate with the upper cell usually enlarged and globose, 8-9,5 x 3-5 $\mu \mathrm{m}$ [COPPINS, 1983].

General distribution: In Europe it has dispersed distribution with limited by few locations from Norway, Finland, and Portugal, Italy to Russia. 


\section{Cladonia norvegica Tønsberg \& Holien}

Description: TøNSBERG, HOLIEN, 1984.

Distribution in Ukraine: Zakarpatska oblast, Velykoberezny district, Volosyanske forestry, 'Adamiv Forest', distr. 22 (area 1), SW of Tykhy village, plantation of Abies, 600$850 \mathrm{~m}$ alt., on wood, 2.06.1998, KS, BJC, PAW, JH, RAA, LAA (9836) (KW).

This species is closely related to Cladonia macilenta and differs by thalli with finely divided basal squamules with a reddish medulla and superficial red spots, $\mathrm{K}+$ purpule [PURVIS et al., 1992].

General distribution: Europe (Norway, Sweden, Finland, Great Britain, Austria, Switzerland, Italy, Czech Republic, Poland, Lithuania, and Slovenia), Madeira, Asia (Russia, Japan), North America (Alaska, Washington, British Columbia, Newfoundland).

\section{Diploschistes gypsaceus (Ach.) Zahlbr.}

Description: LuMBSCH, 1989.

Distribution in Ukraine: Zakarpatska oblast, Velykoberezny district, Velykoberezny collective farm forestry, Mt Stinka, $1057 \mathrm{~m}$ alt., $49^{\circ} 00 \mathrm{~N}^{\prime}, 22^{\circ} 31 \mathrm{E}^{\prime}$, near the border with Slovakia, at the forest edge, on sandstone, 5.08.1997, BJC, $C A, K A$ (loc. 9757) (KW). AR Crimea [ХодОСОвцЕВ, 1999].

This species grows in shaded situation on vertical calciferous rocks and is characterized by densely pruinose greyish white thallus with urceolate apothecia and colourless hypothecium.

General distribution: It has cosmopolite distribution.

\section{Eopyrenula avellanae Coppins}

Description: COPPINS et al. 1992

Distribution in Ukraine: Zakarpatska oblast, Velykoberezny district, Kostrino forestry, distr. 21, area 2, botanical reserve, 'German' Stream, at the bottom of valley, on Corylus avellanae, $48^{\circ} 55^{\prime} \mathrm{N}, 22^{\circ} 34^{\prime} \mathrm{E}$, alt. $350 \mathrm{~m}, B J C, K S$ et al., 29.07.1997 (loc. 9742) (E).

Recently described species with involucrellum outwardly spreading and 3-septate spores. Easily mistaken in the field for Arthopyrenia species or Pyrenula coryli. The latter has ascospores with markedly lens-shaped lumina [PURVIS et al., 1992].

General distribution: In Europe was hitherto collected from W. Britain, N. Ireland and France.

\section{Fellhanera subtilis (Vězda) Diederich \& Sérusiaux}

Description: VĚZDA, 1986.

Distribution in Ukraine: Zakarpatska oblast, Velykoberezny district, Novostuzhytzia forestry, 'Yasynny' ridge, $1060 \mathrm{~m}$ alt., $49^{\circ} 05 \mathrm{~N}^{\prime}, 22^{\circ} 34 \mathrm{E}$ ', old sycamore-beech forest with Lobaria, on Fagus, 1.08.1997, BJC, KS, PAW \& ZSD (tag 2029) (E, KW).

The species was collected with picnidia only. Differs from sterile Bacidia species by picnidia with gapping ostiole and pyriform conidia 3-4 x 1,3-1,7 $\mu \mathrm{m}$. It was collected together with Fellhanera gyrophorica with K+ (purple) picnidia [SÉRUSIAUX et al., 2001].

General distribution: Europe (Norway, Finland, Denmark, Belgium, Netherlands, Poland, Lithuania, Italy), North America).

\section{Fuscidea arboricola Coppins \& Tønsberg}

Description: TøNSBERG, 1992; PURVIS et al., 1992.

Distribution in Ukraine: Zakarpatska oblast, Velykoberezny district, Kostrino forestry, distr. 21, area 2, botanical reserve, 'German' Stream, at the bottom of valley, on Abies, on Fagus, $48^{\circ} 55^{\prime} \mathrm{N}, 22^{\circ} 34^{\prime} \mathrm{E}$, c. $350 \mathrm{~m}, 29.07 .1997, K S$, BJC et al. (loc. 9742) (E); Novostuzhytzia forestry, 'Kamyanysty' ridge, 910-960 m alt., 49 $04^{\circ} \mathrm{N}, 22^{\circ} 36^{\prime} \mathrm{E}$, sycamorebeech forest with Lobaria, on Acer pseudoplatanus, 30.07.1997, KS, BJC, PAW (loc. 9744, 
tag 2022) (E); Stavne forestry, distr. 26, area 1, in the upper part of ridge near Lyskovets Stream, on Fagus, 3.08.97, KA, CA, ZSD (loc. 9749) (E); Novostuzhytzia forestry, ridge unnamed between 'Sukha Potochyna' and 'Husariv' Sreams, distr. 15 (13), on Fagus, 27.09.1997, BJC, KS et al. (tags 2072) (KW). AR Crimea [CopPINS et al., 2001].

This sorediate species has discrete and delimited soralia at thallus edges, which from $\mathrm{Pd}+$ becoming red.

General distribution: The species was hitherto collected from Europe (Scandinavia, Iceland, North Scotland, Switzerland, Austria, Slovenia, Slovakia, Lithuania), North America.

\section{Fuscidea cyatoides (Ach.) V. Wirth \& Vězda var. corticola (Fr.) Kalb}

Description: PURVIS et al., 1992.

Distribution in Ukraine: Zakarpatska oblast, Velykoberezny district, Novostuzhytzia forestry, 'Kamyanysty' stream, distr. 4 (?), 580 m alt., on Fagus, 6.10.1997, KS, ZSD (loc. 97107) (KW).

It differs from Fuscidea arboricola by the rimose-cracked, rarely sorediate thallus with apothecia.

General distribution: Europe (Norway, Denmark, Spain, Czech Republic), Asia (Turkey, Japan).

\section{Fuscidea pusilla Tønsberg}

Description: TøNSBERG, 1992.

Distribution in Ukraine: Zakarpatska oblast, Velykoberezny district, Novostuzhytzia forestry, distr. 2, area 10, near Mt Semenova, sycamore-beech forest with Lobaria, on Fagus, 28.07.1997, KS, BJC et al. (tag. 2002, loc. 9741) (E, KW); distr. 5, area 3, Mt Cheremkha, alt. $1060 \mathrm{~m}$ alt., $49^{\circ} 02^{\prime} \mathrm{N}, 22^{\circ} 41^{\prime} \mathrm{E}$, old beech forest at NE slope to village Lyuta side with Lobaria, on Fagus, 4.08.1997, KS, BJC et al. (E, KW); vicinity of village Zhornava, 'Parashynsky' Stream, Zhornava forestry, distr. 4, on Fagus, 1.10.1997, KS, KA, ZSD (2092) (KW); Stavne forestry, distr. 26, area 1, in the upper part of ridge near Lyskovets stream, on Fagus, 3.08.97, KS, BJC, ZSD, PAW(E).

This is sterile sorediate crustose lichen with similar morphology to Ropalospora viridis, but this species is usually much lager and more intensely green [TøNSBERG, 1992]. The TLC need for correct identification. There is divaricatic acid present in Fuscidea pusilla, in contrast to perlatolic acid in Ropalospora viridis.

General distribution: Europe (Spain, Switzerland, Austria, Slovenia, Poland, Estonia, Lithuania), North America.

\section{Ropalospora viridis (Tønsberg) Tønsberg}

Syn.: Fuscidea viridis Tønsberg

Description: TøNSBERG, 1992; PURVIS et al., 1992.

Distribution in Ukraine: Zakarpatska oblast, Velykoberezny district, Uzhok pass, near the border with Lviv oblast, Picea forest with young Carpinus betulus, alt. 900 m, on Fagus sylvatyca, 27.07.1997, KS, BJC et al. (loc. 9737) (KW); Novostuzhytzia forestry (the former Stavne forestry) distr. 5, area 3, Mt Cheremkha, $1060 \mathrm{~m}$ alt., $49^{\circ} 02 \mathrm{~N}^{\prime}, 22^{\circ} 41 \mathrm{E}^{\prime}$, old beech forest at NE slope to village Lyuta with Lobaria, on Fagus, 4.08.1997, KS, BJC et al. (tag 2031) (KW); vicinity of Stuzhytzia village, 'Zhyduvsky' Stream, Velykoberezny collective farm forestry, distr. 7, on Fagus, 29.09.1997, KS, BJC et al. (tag 2084) (KW); vicinity of village Zhornava, 'Parashynsky' Stream, Zhornava forestry, distr. 4, on Fagus, 1.10.1997, KS, $K A, Z S D$ (tag 2092); vicinity of village Zhornava, location 'Holanya', Zhornava forestry, distr. 10, on Fagus, 4.10.1997, KS, BJC et al. (KW) (tag 2127); Vicinity of village Zhornava, 'Parashynsky' Stream, Zhornava forestry, distr. 4, $375 \mathrm{~m}$ alt. 1.10.1997, KS, KA, ZSD (tag 2092) (KW); Stuzhansky ridge, frontier posts 68 and 69, 1070-1160 m alt., 25.06.1998, KS, $M O G, T L O, I K(9856$, tag 2217) (KW). 
Material collected in Ukraine was sterile. This is very characteristic species with greyish green thallus (10-20 mm diam.), distinct brown prothallus, green soralia and negative chemical tests (Pd-, K-, C, -, KC-).

General distribution: Europe (Norway, Sweden, Netherlands, Germany, Portugal, Poland, Lithuania, Estonia, Slovenia), North America.

\section{Hypocenomice caradocensis (Leighton ex Nyl.) P. James \& G. Schned.}

Description: TIMDAL, 1984.

Distribution in Ukraine: Zakarpatska oblast, Velykoberezny district, Uzhok pass, near the border with Lviv oblast, Picea forest with young Carpinus betulus, on stump, 27.07.1997, $K S, B J C$ et al. (loc. 9737) (KW); Kostrino forestry, distr. 21, 'Yavornyk' ridge, at the upper timberline, sycamore-beech forest with Lobaria, on stump, 26.09.1997, KS, KA, ZSD (KW).

This is a esorediate crustose sterile lichen. It differs from the non-sorediate Hypocenomice praestabilis by squamulose thallus and negative tests on $\mathrm{K}, \mathrm{C}$ and $\mathrm{Pd}$.

General distribution: It is known from atlantic-subatlantic Europe (Portugal, Spain, Germany, Lithuania), Alps (Slovenia) and Carpathians (Czech Republic, Slovakia).

\section{Hypocenomyce xanthococca (Sommerf.) P. James \& G. Schneider}

Description: TIMDAL, 1984.

Distribution in Ukraine: Zakarpatska oblast, Velykoberezny district, Uzhok pass, near the border with Lviv oblast, Picea forest with young Carpinus betulus, on stump, 27.07.1997, $K S$, BJC et al. (KW).

General distribution: Europe.

\section{Jamesiella anastomosans (P. James \& Vězda) Lücking, Sérusiaux \& Vězda}

Syn.: Gyalideopsis anastomosans P. James \& Vězda

Description: PURVIS et al., 1992, LÜCKING et al., 2005.

Distribution in Ukraine: Zakarpatska oblast, Velykoberezny district, Kostrino forestry, distr. 21, area 2, botanical reserve, 'German' Stream, at the bottom of valley, on young trees by stream, $48^{\circ} 55^{\prime} \mathrm{N}, 22^{\circ} 34^{\prime} \mathrm{E}$, c. $350 \mathrm{~m}, 29.07 .1997, K S, B J C$ et al. (loc. 9742) (E).

The presence a thin hyphophores to $0,1 \mathrm{~mm}$ long on filmy, continuous, skin-like thallus are main distinguishing characters from other European species.

General distribution: Europe (British Isles, Spain, France, Belgium, Norway, Lithuania), Canary Islands.

\section{Lecania cyrtellina (Ach.) Th. Fr.}

Description: PURVIS et al., 1992.

Distribution in Ukraine: Zakarpatska oblast, Velykoberezny district, Novostuzhytzia forestry, 'Kamyanysty' ridge, 910-960 m alt., $49^{\circ} 04^{\prime} \mathrm{N}, 22^{\circ} 36^{\prime} \mathrm{E}$, sycamore-beech forest with Lobaria, on Acer pseudoplatanus, 30.07.1997, KS, BJC, PAW (E, KW) (loc. 9744, tag 2021); Novostuzhytzia forestry, 'Yasynny' ridge, $1060 \mathrm{~m}$ alt., $49^{\circ} 05^{\prime} \mathrm{N}, 22^{\circ} 34^{\prime} \mathrm{E}$, old sycamore-beech forest with Lobaria, on Acer, 1.08.1997, KS, BJC, PAW, ZSD (E, KW); near Stavne village, $48^{\circ} 59^{\prime} \mathrm{N}, 22^{\circ} 43^{\prime} \mathrm{E}$, alt. $350 \mathrm{~m}$, on Acer pseudoplatanus, 27.07.1997, KS, BJC et al. (E).

The species is closely related to Lecania cyrtella, but distinguishing by much narrower ascospores and not association with Xanthorion communities.

General distribution: It has local distribution in Europe (Belgium, Poland, Slovakia, Slovenia, Majorca), Canary Islands and North America.

\section{Lecanora cinereofusca Magnusson}

Non Lecanora cinereofusca Motyka

Description: PURVIS et al., 1992. 
Distribution in Ukraine: Zakarpatska oblast, Velykoberezny district, Novostuzhytzia forestry, at the bottom of the valley, Streams 'Bystry' et 'Kamyanysty', distr. 5/6, on Fagus, 01.08.1997, CA, KA (KW) (near tag 2041).

This species can often be separated from L. pulicaris by the more orange apothecial discs and $\mathrm{Pd}+$ orange-red epithecium.

General distribution: Europe (Norway, Italy, Slovakia), Caucasus (Russia), Asia (Turkey, India [as Lecanora cinereofusca var. hymalayensis]) and North America (as Lecanora cinereofusca var. appalachensis).

\section{Lecanora farinaria Borrer in Hook.}

Description: PURVIS et al., 1992.

Distribution in Ukraine: Zakarpatska oblast, Velykoberezny district, S of Kostrino village, 'Yasynny' ridge, a. $1000 \mathrm{~m}$ alt., 26.09.1997, KS, KA, ZSD (9834) (KW).

This sorediate species characteristic by blackish apothecial discs, thick, sorediate exiple and thallus with soralia $\mathrm{K}+$ yellow. Kong).

General distribution: Europe (Norway, Austria, Italy), North America, Asia (Hong

\section{Lecidea swartzioidea Nyl.}

Description: BRODO 1995.

Distribution in Ukraine: Zakarpatska oblast, Velykoberezny district, Novostuzhytzia forestry distr. 5, Mt Cheremkha, on siliceous rock, 2.08.1997, BJC, KS et al. (9752) (E).

General distribution: Europe, North America and New Zealand.

\section{Lepraria lobificans Nyl.}

Description: LAUNDON, 1992; TøNSBERG, 1992.

Distribution in Ukraine: Zakarpatska oblast, Velykoberezny district, Novostuzhytzia forestry (the former Stavne forestry) distr. 5, area 3, Mt Cheremkha, a. $1060 \mathrm{~m}$ alt., 4902N', $22^{\circ} 41 E^{\prime}$, old beech forest at NE slope to village Lyuta side with Lobaria, on bark of Fagus sylvatyca, 4.08.1997, KS, BJC et al. (tags 2032, 2039) (SW of tag 2056) (E); Novostuzhytzia forestry, 'Yasynny' ridge, $1060 \mathrm{~m}$ alt., $49^{\circ} 05 \mathrm{~N}$ ', $22^{\circ} 34^{\prime} \mathrm{E}$, old sycamore-beech forest with Lobaria, on Fagus, 1.08.1997, KS, BJC, WP, ZSD (SW of 2036); Kostrino forestry, distr. 21, area 2, botanical reserve, "German" Stream, at the bottom of valley, on bark of Corylus avellanae, $48^{\circ} 55^{\prime} \mathrm{N}, 22^{\circ} 34^{\prime} \mathrm{E}$, alt. $350 \mathrm{~m}, 29.07 .1997, \mathrm{KS}, B J C$ (loc. 9742) (E, KW); areas 20 and 23, 11.07.1998, $K S$ (tag 2249); Bystrytske forestry, between villages Chornoholovka and Lyuta, 7-8 km NE from village Chornoholovka, valley of Lyutyanka River, distr. 22, a. $350 \mathrm{~m}$ alt., on bark of Acer platanoides, 6.06.1998, KS, BJC, PAW, ZSD, RAA, LAA (KW) (tag 2191); distr. 19, a. $290 \mathrm{~m}$ alt., on bark of Fraxinus, 6.06.1998, KS, BJC, $P A W, Z S D, R A A$, $L A A$ (tag 2195) (KW); Lyutyanske forestry, SW of Lyuta village, distr. 15, 'Shyroky' Stream, on bark of Acer pseudoplatanus, 5.06.1998, KS, BJC, PAW, ZSD, RAA, LAA (tag 2182) (KW); Lyutyanske forestry, 'Rakivska yama', SW of Lyuta village, distr. 20, $1050 \mathrm{~m}$ alt., on bark of Acer pceudoplatanus, 4.06.1998, KS, BJC, ZSD, RAA, LAA (tag 2171, loc. 9841); to E of 'Rakivska yama', SW of Lyuta village, distr. 20, 1050-1150 m alt., N, NNE slopes, beechsycamore forest, on bark of Fagus, 4.06.1998, KS (loc. 9843); Lyutyanske forestry, SW of Lyuta village, distr. 18, 'Mashyn' Stream, 615-620 m alt., on bark of Fagus, 5.06.1998, KS, $B J C, P A W, Z S D, R A A, L A A$ (tag 2178); Novostuzhytzia forestry, ridge unnamed between 'Sukha Potochyna' and 'Husariv' Sreams, distr. 15 (13), on Fagus, 27.09.1997, BJC, KS et al. (tags 2066, 2072) (KW); on Fagus, 2.10.1997, KS, KA, ZSD (tags 2105, 2113) (KW); distr. 14 (15), 1000 m alt., on Fagus, 30.05.1998, KS, BJC, PAW, CS, JH, KA, ZSD et al. (tag 2150; loc. 9833); Novostuzhytzia forestry, distr. 2, area 10, near Mt Semenova, sycamore-beech forest with Lobaria, on Acer pseudoplatanus, 28.07.1997, KS et al. (loc. 9741, tag 2008). 
Rather common within area studied species. AR Crimea [ХОдОсовцЕв, 1999, 2000, 2003; ХОДОСОВЦЕВ, РЕДЧЕНКО, 2002, БОГДАН, 2002; ХОДОСОВЦЕВ, БОГДАН, 2005].

The bright, pale green colour of the thallus ( $\mathrm{Pd}+$ orange, $\mathrm{K}-$ or + yellow, $\mathrm{C}-)$ is distinctive for this species. Leproloma vouauxii differs by whitish to pale yellowish-grey thallus (Pd- to + reddish orange, $\mathrm{K}-, \mathrm{C}-$ ).

General distribution: It has cosmopolite distribution. Europe (from Portugal, Denmark, Germany, Italy to Estonia, Croatia and Greece), North America (Canada - British Columbia), Asia (India).

\section{Lepraria rigidula (B. de Lesd.) Tønsberg}

Description: TøNSBERG, 1992.

Distribution in Ukraine: Zakarpatska oblast, Velykoberezny district, Kostrino forestry, distr. 21, area 2, botanical reserve, 'German' Stream, at the bottom of valley, Lobaria, on Sorbus, 29.07.1997, KS, BJC et al. (loc. 9742) (E); Novostuzhytzia forestry, 'Yasynny' ridge, $1060 \mathrm{~m}$ alt., $49^{\circ} 05 \mathrm{~N}^{\prime}, 22^{\circ} 34 \mathrm{E}^{\prime}$, old sycamore-beech forest with Lobaria, on Fagus, 1.08.1997, $K S, B J C, P A W, Z S D$ (loc 9745 tag 2025, loc. 9746, tag. 2029) (E); Novostuzhytzia forestry, at the bottom of the valley, Streams 'Bystry' and 'Kamyanysty', distr. 5, 6, 12, 490-555 m alt. (loc. 9754); Novostuzhytzia forestry, ridge unnamed between 'Sukha Potochyna' and 'Husariv' Sreams, distr. 15 (13), on Fagus, 2.10.1997, KS, KA, ZSD (tag 2109, 2110) (KW); vicinity of Stuzhytzia village, 'Zhyduvsky' Stream, Velykoberezny collective farm forestry, distr. 7, on Fagus, 29.09.1997, KS, KA \& ZSD (KW); distr. 1, 420 m alt., on Fagus, 3.10.1997, KS, KA, ZSD (tag 2116). AR Crimea [ХОДОСОВЦЕВ, БОГДАН, 2005].

It differs from Lepraria lobificans by whitish thallus and negative reaction of thallus with Pd.

General distribution: Europe (from Iceland, Norway and Sweden to southern European countries, Russia, and Bulgaria), Asia (Turkey), North Africa (Morocco), and North America.

\section{Leproloma vouauxii (Hue) J.R. Laundon}

Description: LAUNDON, 1989.

Distribution in Ukraine: Zakarpatska oblast, Velykoberezny district, Novostuzhytzia forestry, 'Yasynny' ridge, $1060 \mathrm{~m}$ alt., $49^{\circ} 05 \mathrm{~N}$ ', $22^{\circ} 34 \mathrm{E}^{\prime}$, old sycamore-beech forest with Lobaria, on Acer pseudoplatanus, 1.08.1997, KS, BJC, PAW, ZSD (E, KW); Novostuzhytzia forestry, distr. 8, area 10, not far from forestry house, beech forest with Lobaria, 19.06.1997, $K S$ (9706); Novostuzhytzia forestry, distr. 2, area 10, near Mt Semenova, sycamore-beech forest with Lobaria, on Acer pseudoplatanus, 28.07.1997, KS, BJC et al. (02001) (E); Novostuzhytzia forestry, ridge unnamed between 'Sukha Potochyna' and 'Husariv' Sreams, distr. 14 (15) or distr. 15 (13), on Fagus, 27.09.1997, KS, KA, ZSD (loc. 9793, tag 2064) (KW). AR Crimea [ХодосовцЕв, 2003].

It is cosmopolitic species, which differs from Leproloma membranacea by obscurely lobed and whitish colour of the thallus.

General distribution: It has sparse localities in Europe (Atlantic part of Europe, Finland, Russia, Estonia, Lithuania).

\section{Leptogium teretiusculum (Wallr.) J.R. Laundon}

Description: JøRGENSEN, JAMES, 1983.

Distribution in Ukraine: Zakarpatska oblast, Velykoberezny district, Novostuzhytzia forestry, 'Yasynny' ridge, $1060 \mathrm{~m}$ alt., $49^{\circ} 05^{\prime} \mathrm{N}, 22^{\circ} 34^{\prime} \mathrm{E}$, old sycamore-beech forest with Lobaria, on Fagus, 1.08.1997, KS, BJC, WP, ZSD (SW of 2036); Novostuzhytzia forestry (the former Stavne forestry) distr. 5, area 3, Mt Cheremkha, $1060 \mathrm{~m}$ alt., 49 $02 \mathrm{~N}^{\prime}, 2^{\circ} 41 \mathrm{E}^{\prime}$, old beech forest, on Fagus, 04.08.97, KS, BJC et al. (E) (SW of 2056, 9752). AR Crimea [ХОДОСОВЦЕВ, РЕДЧЕНКО, 2002]. 
The species is related to Leptogium subtile but differs by thallus consisting erect, crowded, minute cylindrical to coralloid branchlets and rare with apothecia.

General distribution: Europe (from Norway to Estonia and Slovenia), Asia (Eastern Siberia), North America.

\section{Leptorhaphis maggiana (A. Massal.) Korber}

Description: PURVIS et al., 1992.

Distribution in Ukraine: Zakarpatska oblast, Velykoberezny district, Velykoberezny collective farm forestry, Mt Stinka, $1057 \mathrm{~m}$ alt., $49^{\circ} 00^{\prime} \mathrm{N}, 22^{\circ} 31^{\prime} \mathrm{E}$, near the border with Slovakia, at the forest edge, on Corylus avellanae, 5.08.1997, BJC, CA\& KA (E) (9757).

It is non-lichenized fungus which differs from Leptorhaphis epidermalis by 3-septate ascospores, (25-)25-30 x 1,5-2,5 $\mu \mathrm{m}$ and I+ yellowish hymenial gelatine.

General distribution: It is known from several European countries (Great Britain, Belgium, Luxembourg, Germany, Switzerland, Austria, Italy).

\section{Megalaria pulverea (Borrer) Hafellner \& E. Schreiner}

Description: PURVIS et al., 1992.

Distribution in Ukraine: Zakarpatska oblast, Velykoberezny district, Kostrino forestry, distr. 21, area 2, botanical reserve, 'German' Stream, at the bottom of valley, Lobaria, on Alnus, 29.07.1997, KS, BJC et al. (KW, E) (loc. 9742); areas 20 and 23, 11.07.1998, KS (tag 2249); Novostuzhytzia forestry, at the bottom of the valley, Streams 'Bystry' and 'Kamyanysty', distr. 5/6, on Fagus, 01.08.97, CA, KA (KW) (near tag 2041); distr. 5, area 3, Mt Cheremkha, a. $1060 \mathrm{~m}$ alt., $49^{\circ} 02^{\prime} \mathrm{N}, 22^{\circ} 41^{\prime} \mathrm{E}$, old beech forest at NE slope to village Lyuta side with Lobaria, $970 \mathrm{~m}$ alt., on Fagus, 4.08.1997, KS et al. (02032) (KW); between Stuzhytzia and Zhornava villages, between 'Chorni Mlaky' and 'Divcha' Ridges, Zhornavsky forestry, distr. 25 (29) in the upper part of ridge, $1020 \mathrm{~m}$ alt., on Fagus, 28.09.1997, KS, KA, ZSD (02076). AR Crimea [ХОДОСОВЦЕВ, БОГДАН, 2005].

The sterile specimens of this species superficially can be confused with Mycoblastus sterilis, but distinguished by much thicker grey to glaucous thallus and large soredia (40-100 $\mu \mathrm{m}$ diam.).

(Tasmania).

General distribution: Europe, Macaronesia, South America (Chile), Australia

\section{Micarea adnata Coppins}

Description: COPPINS, 1983.

Distribution in Ukraine: Zakarpatska oblast, Velykoberezny district, vicinity of village Zhornava, 'Zhornavsky' Stream, Zhornava forestry, distr. 9 or distr. 5 (?) 4.10.1997, on old stump, 4.10.1997, KS, ZSD (97103).

It is species easily recognised by presence of white or pallid sporodochia, which resemble small apothecia.

General distribution: The distribution in Europe (Norway, Great Britain, France, Spain, Netherlands, Germany, Switzerland, Austria, Poland, Czech Republic) and Asia (Siberia) close related with high annual precipitation more them 1000 per year.

\section{Micarea hedlundii Coppins}

Description: COPPINS, 1983.

Distribution in Ukraine: Zakarpatska oblast, Velykoberezny district, Volosyanske forestry, 'Adamiv Forest', distr. 22 (area 1), SW of Tykhy village, plantation of Abies, on coniferous stump, 600-850 m alt. 2.06.1998, KS, BJC, PAW, JH, RAA, LAA (9836) (E).

This species is closely related with Micarea prasina agg., but it is easily recognized by distinctly stalked, pinkish brown, tomentose pycnidia. 
General distribution: It is known from scattered localities in Europe (Norway, Sweden, Germany, Czech Republic, Switzerland, Austria, Slovenia, Lithuania, Poland, Slovakia), and North America.

\section{Micarea melaeniza Hedl.}

Description: COPPINS, 1983.

Distribution in Ukraine: Zakarpatska oblast, Velykoberezny district, Volosyanske forestry, 'Adamiv Forest', distr. 22 (area 1), SW of Tykhy village, plantation of Abies, 600$850 \mathrm{~m}$ alt., on coniferous trunk, 2.06.1998, KS, BJC, PAW, JH, RAA, LAA (9836) (E).

This is difficult for identification species, which can be recognized by black, subglobose to tuberculate apithecia, black, stalked pycnidia, inconspicuous thallus, and occurence on lignum. It is closely related with Micarea nigella, M. misella, M. botryoides and M. muhrii. The other characters of these species see in CopPINS [1983].

General distribution: Micarea melaniza was hitherto known only from Sweden and Austria.

\section{Micarea nigella Coppins}

Description: COPPINS, 1983.

Distribution in Ukraine: Zakarpatska oblast, Velykoberezny district, Volosyanske forestry, 'Adamiv Forest', distr. 22 (area 1), SW of Tykhy village, plantation of Abies, on conifeous lignum, 600-850 m alt. 2.06.1998, KS, BJC, PAW, JH, RAA, LAA (9836) (E).

This lignicolous species is characterized by the purple-brown $\mathrm{K}+$ green pigment in the hymenium, hypothecium and pycnidial tissues, simple spores and stalked pycnidia [CoPPINS, 1983].

General distribution: Distribution of this species is limited by Scotland, England, Denmark, Belgium, Luxembourg, France, Czech Republic, Poland and Lithuania.

\section{Micarea peliocarpa (Anzi) Coppins \& R. Sant.}

Description: COPPINS, 1983.

Distribution in Ukraine: Zakarpatska oblast, Velykoberezny district, vicinity of the village Stavne, old beech forest, alt. $350,48^{\circ} 66^{\prime} \mathrm{N}, 22^{\circ} 43^{\prime} \mathrm{E}$, on Acer campestre, 27.07.1997, $K S$, BJC et al. (E); between villages Stavne and Lubnya, Stavne forestry, distr. 12, 'Ertashy' Stream, $680 \mathrm{~m}$ alt., on Acer, 5.10.1997, KS, ZSD (97105); Novostuzhytzia forestry, 'Kamyanysty' stream, distr. 4 (?), $580 \mathrm{~m}$ alt. 6.10.1997, KS, ZSD (97102); Kostrino forestry, distr. 21, area 2, botanical reserve, 'German' Stream, at the bottom of valley, Lobaria on sycamore, on Fagus, 29.06.1997, KS, BJC (9742).

This species closely related with Micarea cinerea and superficially can be mistaken with Micaria leprosula, Micarea nitschkeana and Bacidia naegelii [CopPINS, 1983].

General distribution: Europe (from Iceland to Estonia, Lithuania), Azores, Canary Islands, North America (north-eastern USA, eastern Canada), Asia (Turkey, Hong Kong) and New Zealand.

\section{Mycoblastus sterilis Coppins \& P. James}

Description: COPPINS, JAMES, 1979.

Distribution in Ukraine: Zakarpatska oblast, Velykoberezny district, Uzhok pass, near the border with Lviv oblast, Picea forest with young Carpinus betulus, 900 m., on Fagus, parasitized by Tremella lichenicola, 27.07.1997 KS, BJC et al. (loc. 9737) (KW); Kostrino forestry, distr. 21, area 2, botanical reserve, 'German' Stream, at the bottom of valley, Lobaria on sycamore, $800 \mathrm{~m}$ alt., on Fagus, 29.07.1997, KS, BJC et al. (loc. 9742, near tag 2014) (E); Lyutyanske forestry, SW of Lyuta village, distr. 18, 'Mashyn' Stream, 615-620 m alt., Acer pseudoplatanus, 5.06.1998, KS, BJC, PAW, ZSD, RAA, LAA (tag 2179); Stuzhansky ridge, frontier posts 68 and 69, 1070-1160 m alt., 25.06.1998, KS, MOG, TLO, IK (tag 2217) 
(KW); Novostuzhytzia forestry (the former Stavne forestry) distr. 5, area 3, Mt Cheremkha, a. $1060 \mathrm{~m}$ alt., $49^{\circ} 02^{\prime} \mathrm{N}, 22^{\circ} 41^{\prime} \mathrm{E}$, old beech forest at NE slope to village Lyuta side with Lobaria, on Fagus, 970 m alt., 4.08.1997, KS et al. (KW) (9752).

It is closely related to Mycoblastus fucatus, but distinguished by well-developed prothallus, more or less convex soralia and growing on bark.

General distribution: Northern, Western and Central Europe.

\section{Microcalicium ahlneri Tibell}

Description: TIBELL, 1978.

Distribution in Ukraine: Zakarpatska oblast, Velykoberezny district, Volosyanske forestry, 'Adamiv Forest', distr. 22 (area 1), SW of Tykhy village, plantation of Abies, 600$850 \mathrm{~m}$ alt., 2.06.1998, KS, BJC, PAW, JH, RAA, LAA (9836) (KW).

General distribution: Europe (Germany, Czech Republic, Italy), Asia (Russia - Kuril Islands, China).

\section{Ochrolechia szatalensis Verseghy}

Description: PURVIS et al., 1992.

Distribution in Ukraine: Lyuta, without details on locality (E).

This species connected with Ochrolechia parella, but distinguished by apothecial disc pruina $\mathrm{C}+$ yellow, in contrast $O$. parella has $\mathrm{C}+$ red reactions.

General distribution: It is known from Great Britain, Italy and Norway.

General distribution: Europe, North Africa (Morocco).

\section{Pertusaria pupillaris (Nyl.) Th. Fr.}

Description: PURVIS et al., 1992.

Distribution in Ukraine: Zakarpatska oblast, Velykoberezny district, Novostuzhytzia forestry, 'Kamyanysty' ridge, 910-960 m alt., 49 $044^{\circ} \mathrm{N}, 22^{\circ} 36^{\prime} \mathrm{E}$, sycamore-beech forest with Lobaria, on Corylus avellanae, 30.07.1997, KS, BJC, WP (KW); Between Stuzhytzia and Zhornava villages, between 'Chorni Mlaky' and 'Divcha' Ridges, Zhornavsky forestry, distr. 25 (29) in the upper part of ridge, 1020 m alt., on Fagus, 28.09.1997, KS, KA, ZSD (02076). AR Crimea [ХОДОСОВЦЕВ, БОГДАН, 2005].

It superficially resembles young thalli of Phlyctis, but distinguished by small, punctiform $\mathrm{Pd}+$ orange-red soralia, $\mathrm{K}+$ dirty reddish thallus and Lecanora-like apothecia if it's fertile.

General distribution: Europe (from Fennoscandia, British Islands and Pyrenees to Czech Republic, Lithuania and Slovenia), North America.

\section{Phaeophyscia endophoenicea (Harm.) Moberg}

Description: MOBERG, 1977.

Distribution in Ukraine: Zakarpatska oblast, Velykoberezny district, vicinity of village Zhornava, near farm building on NE vicinity of the village (from Stavne side), on Acer, 27.07.1997, KS, BJC et al. (9740) (KW); Novostuzhytzia forestry (the former Stavne forestry) distr. 5, area 3, Mt Cheremkha, a. $1060 \mathrm{~m}$ alt., $49^{\circ} 02^{\prime} \mathrm{N}, 22^{\circ} 41^{\prime} \mathrm{E}$, old beech forest at NE slope to village Lyuta side with Lobaria, on Fagus, 4.08.1997, KS, BJC et al. (loc. 9752, tag 2032, 2033, 2037) (E, KW); Novostuzhytzia forestry, at the bottom of the valley, Stream 'SemenivSokoliv', 49 $03^{\circ} \mathrm{N}, 22^{\circ} 35^{\prime} \mathrm{E}$, a. $470 \mathrm{~m}$ alt., on Fraxinus, 30.07.1997, CA et al. (loc. 9744); on Carpinus betulis by stream, CA et al., and on Fagus, 30.07.1997, BJC et al. (loc. 9743, tag 2015) (E, KW); Novostuzhytzia forestry, 'Yasynny' ridge, $1020 \mathrm{~m}$ alt., old beech tree with Lobaria in young beech forest, on Fagus, 1.08.1997, BJC, BJC et al. (loc. 9745, tag 2025) (KW); 'Yasynny' ridge, $1060 \mathrm{~m}$ alt., $49^{\circ} 05 \mathrm{~N}^{\prime}, 22^{\circ} 34^{\prime} \mathrm{E}$, old sycamore-beech forest with Lobaria, on Fagus, 1.08.1997, KS, BJC, PAW, ZSD (loc 9745 tag 2025, loc. 9746, tag. 2029) (E); Novostuzhytzia forestry, ridge unnamed between 'Sukha Potochyna' and 'Husariv' 
Sreams, distr. 14 (15) or distr. 15 (13), on Fagus, 27.09.1997, KS, KA, ZSD (loc. 9793, tag 2064) (KW); 1000 m alt., on Acer platanoides, 30.05.1998, KS, BJC, PAW, CS, JH, KA, ZSD et al. (tag 2152). AR Crimea [ХодОсОВцЕВ, 2000].

A medulla of this species is orange, $\mathrm{K}+$ purple, while related Phaeophyscia orbicularis usually has white medulla, but sometimes yellow to orange only in uppermost part of cortex.

General distribution: Europe (from Central and North countries to Lithuania and Bulgaria), Asia (Turkey).

\section{Phaeophyscia hirsuta (Mereschk.) Moberg}

Description: MOBERG, 1977; NOWAK, 1994.

Distribution in Ukraine: vicinity of the village Kostrino, on Cerasus vulgaris, 4.06.1998, CS (KW).

The hyaline hairs on upper cortex and terminal lip-shaped soredia are distinguished from Phaeophyscia orbicularis.

General distribution: Europe, Asia (Mongolia), North (Morocco), East (Kenya) and South Africa, North (Canada, USA, Mexico), and South America (Argentina).

\section{Physcia vitii Nadv.}

Description: NowAK, 1994.

Distribution in Ukraine: Zakarpatska oblast, Velykoberezny district, vicinity of the village Kostrino, on Cerasus vulgaris, 4.06.1998, CS (KW).

The pseudoparenchimatous lower cortex, lip-like soralia and lake cilia are characteristic and distinguished from Physcia dubia and Physcia adscendens agg. The closely related Physcia tribacia has marginal soralia and grows mostly on rock.

General distribution: The distribution of Physcia vitii is unstudied and it is hitherto known only from Italy, Switzerland, Slovakia and Poland.

\section{Porina leptalea (Durieu \& Mont.) A.L. Sm}

Description: PURVIS et al., 1992.

Distribution in Ukraine: Zakarpatska oblast, Velykoberezny district, Kostrino forestry, distr. 21, area 2, botanical reserve, 'German' Stream, at the bottom of valley, Lobaria on sycamore, on Fagus by stream crossing, $48^{\circ} 55^{\prime} \mathrm{N}, 22^{\circ} 34^{\prime} \mathrm{E}$, c. 350 m, 29.07.1997, KS, BJC (loc. 9742) (KW).

The orange to red-brown perithecia are the main characters of this species.

General distribution: This species has a southern and western distribution in Europe, extending as far north as Finland, and has also been reported from North America [NIMIS, 1993].

\section{Ramonia chrysophaea (Pers.) Vězda}

Description: COPPINS, 1987 b.

Distribution in Ukraine: Zakarpatska oblast, Velykoberezny district, Novostuzhytzia forestry, distr. 2, area 10, near Mt Semenova, sycamore-beech forest with Lobaria, Acer pseudoplatanus, 28.07.1997, KS, BJC (loc. 9741, tag 2001) (KW).

This genus differs from Gyalecta or Pachyphiale in having a true exciple composed of narrow, never distinctly angular, cells and lacking periphyses [PURVIS et al., 1992]. Ramonia chrisophaea has needle-shaped ascospores mostly more $45 \mu \mathrm{m}$ long 8- to 14-septate. The specimen was with one apothecium only which was destroyed during identification.

General distribution: It is known from British Isles, Denmark, France and Spain. 
Distribution in Ukraine: Zakarpatska oblast, Velykoberezny district, Kostrino forestry, distr. 21, area 2, botanical reserve, 'German' Stream, at the bottom of valley, Lobaria on sycamore, on Acer pseudoplatanus, 29.07.1997, KS, BJC et al. (loc. 9742, tag 2013) (E, KW); areas 20 and 23, 11.07.1998, KS (tag 2249); vicinity of Stuzhytzia village, 'Zhyduvsky' Stream, Velykoberezny collective farm forestry, distr. 7, on Acer pseudoplatanus, 29.09.1997, $K S$ et al. (loc. 9795, 9796, tags 2083, 2087) (KW); vicinity of village Zhornava, 'Parashynsky' Stream, Zhornava forestry, distr. 4, on Carpinus betulus, 1.10.1997, KS et al. (loc. 9799, tag 2102) (KW); vicinity of Stuzhytzia village, 'Chorny' Stream, Velykoberezny collective farm forestry, distr. 1, on Fagus, 3.10.1997, KS et al. (loc. 97102, tag 2116) (KW); Lyutyanske forestry, SW of Lyuta village, distr. 15, 'Shyroky' Stream, on Fraxinus, 5.06.1998, KS, BJC, PAW, ZSD, RAA, LAA (02186); Novostuzhytzia forestry, ridge 'unnamed' between 'Sukha Potochyna' and 'Husariv' Streams, distr. 14 (15) or distr. 15 (13), $1050 \mathrm{~m}$ alt., on Acer pseudoplatanus, 27.09.1997, KS, KA, ZSD (02080).

The genus resembles some species Taeniolella with a verrucose conidial wall, but is easily distinguished by the branched conidia and the tendency of the conidiophore to form sporodochia [DIEDERICH, SCHEIDEGGER, 1996]. Reichlingia leopoldii was described as lichenicolous fungi, but appears to be hyphomycetes Trenthepohlia containing lichenized fungus.

General distribution: British Isles, Central Europe, Poland, and Lithuania.

\section{Rinodina griseosoralifera Coppins}

Description: COPPINS, 1989.

Distribution in Ukraine: Zakarpatska oblast, Velykoberezny district, Novostuzhytzia forestry, 'Yasynny' ridge, $1060 \mathrm{~m}$ alt., $49^{\circ} 05^{\prime} \mathrm{N}, 22^{\circ} 34^{\prime} \mathrm{E}$, old sycamore-beech forest with Lobaria, on Fagus, 1.08.1997, KS, BJC et al. (near tag 2026) (KW); Novostuzhytzia forestry, ridge unnamed between 'Sukha Potochyna' and 'Husariv' Streams, distr. 14 (15), $1000 \mathrm{~m}$ alt., on Acer platanoides, 30.05.1998, KS, BJC, PAW, CS, JH, KA, ZSD et al. (tag 2152).

It is sorediate species with blue-grey soredia, which is in Pd negative or yellowish. $R$. efflorescens is closely related to $R$. griseosoralifera, but has $\mathrm{Pd}+$ (orange) soredia reaction.

General distribution: Europe (Norway, Scotland, England, Wales, Austria, Switzerland, Czech Republic), Africa (Canary Islands), and North America.

\section{Rinodina efflorescens Malme}

Description: PURVIS et al., 1992.

Distribution in Ukraine: Zakarpatska oblast, Velykoberezny district, Novostuzhytzia forestry, 'Yasynny' ridge, $1170 \mathrm{~m}$ alt., $49^{\circ} 05^{\prime} \mathrm{N}, 22^{\circ} 34^{\prime} \mathrm{E}$, old sycamore-beech forest with Lobaria, on Acer pseudoplatanus, 1.08.1997, BJC (loc. 9746) (E), on Fagus, 1.08.1997, KS, $B J C, P A W, Z S D$ (loc 9745) (KW); Novostuzhytzia forestry, at the bottom of the valley, Streams 'Bystry' and 'Kamyanysty', distr. 5, 6, 12, 490-555 m alt., 1.08.1997, $K A, C A$ (9754).

It is separated from $R$. griseosoralifera with its $\mathrm{Pd}+$ yellow but never $\mathrm{Pd}+$ (orange) reaction [PURVIS et al., 1992].

General distribution: Europe (Norway, Sweden, British Isles, Belgium, Germany, Poland, Lithuania, Estonia, Portugal), Asia (Turkey) and North America (Canada).

\section{Telocarpon strasseri Zahlbr.}

Description: POELT, 1969.

Distribution in Ukraine: Zakarpatska oblast, Velykoberezny district, Kostrino forestry, distr. 21, area 2, botanical reserve, 'German' Stream, at the bottom of valley, on pebbles, 29.07.1997, KS, BJC et al. (KW).

General distribution: Europe (Belgium, Luxembourg, Netherlands). 
51. Shismatomma ricasolii (A. Massal.) Egea \& Torrente

Description: TORRENTE, EGEA, 1989; TEHLER, 1994.

Distribution in Ukraine: Zakarpatska oblast, Velykoberezny district, Lyuta forestry, area 12, N slopes to Lyuta Stream, 635-700 m, on Acer pseudoplatanus, 1.08.1998, KS, KA, ZSD, LAA, KNS, KOO $(9897,2315)$.

General distribution: Europe.

\section{Trapelia corticola Coppins \& P. James}

Description: COPPINS, JAMES, 1984.

Distribution in Ukraine: Zakarpatska oblast, Velykoberezny district, Stavne forestry, distr. 26, area 1, Lyskovets Sream, at the bottom of valley, on woods, 3.08.1997, $C A(\mathrm{KW})$; Velyky Berezny collective farm forestry, Mt Stinka, $1057 \mathrm{~m}$ alt., $49^{\circ} 00^{\prime} \mathrm{N}, 22^{\circ} 31^{\prime} \mathrm{E}$, near the border with Slovakia, at the forest edge, on Fagus, 5.08.1997, KS, WP, ZSD (9753) (KW); Vicinity of village Zhornava, 'Zhornavsky' Stream, Zhornava forestry, distr. 9 or distr. 5 (?), 380-440 m alt., on Fagus, 4.10.1997, KS, ZSD (97103); Novostuzhytzia forestry, ridge unnamed between 'Sukha Potochyna' and 'Husariv' Streams, distr. 14 (15), $1000 \mathrm{~m}$ alt., on Fagus, 30.05.1998, KS, BJC, PAW, CS, JH, KA, ZSD et al. (02150); Lyutyanske forestry, SW of Lyuta village, distr. 18, 'Mashyn' Stream, 615-620 m alt., on Fagus, 5.06.1998 KS, BJC, $P A W, Z S D, R A A, L A A$ (02177); Volosyanske forestry, 'Adamiv Forest', distr. 22 (area 1), SW of Tykhy village, plantation of Abies, on woods, 600-850 m alt., 2.06.1998, KS, BJC, PAW, $J H, R A A, L A A$ (9836). AR Crimea [РЕДЧЕНКО, 2001].

Whole collection is sterile. The inconspicuous, greenish or brownish areoles, numerous, small, punctiform soralia $(\mathrm{C}+\mathrm{red})$ suggest $T$. corticola is related to $T$. obtegens, but these species have different ecology.

General distribution: Atlantic Europe (British Isles, Spain), Tuscany, Azores, Macaronesia, North America (British Columbia), and South America (Chile).

\section{Trapeliopsis pseudogranulosa Coppins \& P. James}

Description: COPPINS, JAMES, 1984.

Distribution in Ukraine: Zakarpatska oblast, Velykoberezny district, Kostrino forestry, distr. 21, area 2, botanical reserve, 'German' Stream, at the bottom of valley, on woods, $48^{\circ} 55^{\prime} \mathrm{N}, 22^{\circ} 34^{\prime} \mathrm{E}$, c. $350 \mathrm{~m}, 25.06 .1997, K S, B J C$ (loc. 9742) (KW); Volosyanske forestry, 'Adamiv Forest', distr. 22 (area 1), SW of Tykhy village, plantation of Abies, on woods, 600850 m alt., 2.06.1998, KS, BJC, PAW, JH, RAA, LAA (9836).

It is characterized by its minutely granular thallus, $\mathrm{C}+$ red soralia and irregular, patchy coloration ( $\mathrm{K}+$ purple) due to the presence of an antraquinone [COPPINS, JAMES, 1984].

General distribution: This species is hitherto common and widely distributed in Europe (from the British Isles, Portugal to Czech Republic, Poland, Estonia), Madeira, Canary Islands, North America, South America (Chile), New Zealand.

\section{Vezdaea aestivalis (Ohl.) Tsch.-Woess \& Poelt}

Description: TsCHERMAK-WoEss, POELT, 1976; COPPINS, 1987 a.

Distribution in Ukraine: Zakarpatska oblast, Velykoberezny district, Novostuzhytzia forestry, at the bottom of the valley, Streams 'Bystry' and 'Kamyanysty', distr. 5, $49^{\circ} 05^{\prime} \mathrm{N}$, $22^{\circ} 34^{\prime} \mathrm{E}$, on mousses on Fraxinus, 1.08.1997, KS, CA et al. (E); at the bottom of the valley, Streams 'Bystry' and 'Kamyanysty', distr. 5, 6, 12, 490-555 m alt., 1.08.1997, KA, CA (loc. 9754); Lyutyanske forestry, SW of Lyuta village, distr. 18, 'Mashyn' Stream, 615-620 m alt., on Acer platanoides, 5.06.1998, KS, BJC, PAW, ZSD, RAA, LAA (tags 02176, 2182); Novostuzhytzia forestry (the former Stavne forestry) distr. 5, area 3, Mt Cheremkha, $1060 \mathrm{~m}$ alt., $49^{\circ} 02^{\prime} \mathrm{N}, 22^{\circ} 41^{\prime} \mathrm{E}$, old beech forest at NE slope to village Lyuta with Lobaria, on Fagus, 4.08.1997, KS, BJC et al. (tag 2031) (KW); 
This species resembles superficially Micarea, but short-lived convex tomentose apothecia without exiple and hypothecium are diagnostical characters.

General distribution: Europe (from Denmark and Spain to Poland, Estonia, Russia, Lithuania).

\section{Zamenhofia hibernica (P. James \& Swinscow) Clauz. \& Cl. Roux}

Description: SWINSCOW, 1962, JAMES, 1971.

Distribution in Ukraine: Zakarpatska oblast, Velykoberezny district, Novostuzhytzia forestry (the former Stavne forestry) distr. 5, area 3, Mt Cheremkha, a. $1060 \mathrm{~m}$ alt., $49^{\circ} 02^{\prime} \mathrm{N}$, $22^{\circ} 41^{\prime} \mathrm{E}$, old beech forest at NE slope to village Lyuta side with Lobaria, on Fagus, 4.08.1997, BJC et al. (loc. 9752, tags 2032, 2033, 2037) (E, KW); Novostuzhytzia forestry, at the bottom of the valley, Stream 'Semeniv-Sokoliv', $49^{\circ} 03^{\prime} \mathrm{N}, 22^{\circ} 35^{\prime} \mathrm{E}$, a. $470 \mathrm{~m}$ alt., on Carpinus betulis by stream, CA et al., and on Fagus, 30.07.1997, BJC et al. (loc. 9743, tag 2015) (E, KW); Novostuzhytzia forestry, ridge unnamed between 'Sukha Potochyna' and 'Husariv' Sreams, distr. 15 (13), on Fagus, 2.10.1997, KS et al. (loc. 97100, tag 2105) (KW).

The specimens studied were sterile. Morphologically it is related to sterile Saccomorpha icmalea, but $Z$. hibernica has Trentepohlia algae in coralloid isidia.

General distribution: It grows on sheltered trunks of old ancient woodland in Europe (England, Denmark, Italy, France and Spain).

\section{Acknowledgements}

We are grateful to Director of the Uzhansky N.N.P. Mr Vasyl O. Kopach (Velyky Berezny); Head of Novostuzhytzia forestry Mrs Ganna Yu. Genyuta (Zhornava) for substantial help during field work; to Mrs Lyudmyla Popova and Ms Natalya G. Beznis (Kiev) for support of all kinds during treatment of material collected and preparation of this paper.

The financial support of Darwin Initiative is gratefully acknowledged.

\section{Literature}

АндРИАнов М. К. Климат // В кн.: Природа Украинских Карпат. - Киев, 1968. - С. 87-101.

БОГДАН О.В. Екологічні особливості епіфітних лишайників соснового лісу долини річки Учан-Су (АР Крим) // Еколого-біологічні дослідження на природних та антропогенно-змінених територіях: Матеріали наук. конференції молодих вчених (Кривий Ріг, 13-16 травня 2002 р.). - Кривий Ріг, 2002. - С. 31-34.

КОНДРАТЮК С.Я., КОППІнС Б., ЗЕЛЕНКО С.Д., ХОДОСОВцЕВ С.Д., КОППІнС О.Є., КОППІНС О., УоЛСЛІ П. До вивчення та охорони лишайників угруповання Lobarion на території регіонального парку "Стужиця" //Заповідна справа в Україні, 1998. - 4, 1. - С. 35-50.

РЕдченкО О.О. Лихенофлора горы Кучук-Аю: состав, екология и проблемы охраны // Ученые записки Таврического ун-та. Серия: Биология. - 2001. - Т.14, №1. - С. 180-183.

ХодосовцЕВ О.С. До вивчення екології епілітних лишайників державного заказника "Водоспад ДжурДжур" // Заповідна справа: стан, проблеми, перспективи. - Херсон, 1999. - С. 75-78.

ХодосовЦЕВ О.Є. Нові для Криму та України види лишайників // Укр. ботан. журн. - 2000. - Т. 57, №5. C. 612-615.

ХодосовцЕВ А.Е. Лишайники карстовых обнажений Чатырдага (Крым) // Ботан. журн. - 2002а. - Т. 87 , № $1 .-$ С. $46-56$.

ХодосовЦЕВ О.С. Екологічні індекси лишайників кам'янистих відслонень Карабі-яйли (АР Крим, Україна) // Природничий Альманах. Серія: Біологічні науки. - Вип. 2, № 3. - Херсон, 2002б. - С. 225-239.

Ходосовцев О.С. Нові для України та Криму види лишайників з силікатних відслонень // Укр. ботан. журн. - 2003. - Т. 60, № 1. - С. 70-78.

ХодосовЦЕВ О.С., БОГДАН О.В. Анотований список лишайників Ялтинського гірсько-лісового природного заповідника // Чорн. ботан. журн. - 2005. - Т.1, № 1. - С. 117-132.

ХодосовцЕв О.С., РЕДЧЕНКО О.О. Анотований список лишайників заповідника “Мис Март’ян” // Укр. ботан. журн. - 2002. - Т. 59. № 1. - С. 64-71.

BRodo I.M. Lichens and lichenicolous fungi of the Queen Charlotte Islands, British Columbia, Canada. 1. Introduction and new records for B.C., Canada and North America // Mycotaxon. - 1995. - Vol. 56. P.135-173. 
Coppins B. A taxonomic study of the lichen genus Micarea in Europe // Bull. Br. Mus. nat. Hist. (Bot.). - 1983. - Vol. 11, N 2. - P. 17-214.

Coppins B.J. The genus Vezdaea in the British Isles // Lichenologist. - 1987a. - Vol. 19. - P. 167-181.

CoppIns B.J. The genus Ramonia in the British Isles // Lichenologist. - 19876. - Vol. 19. - P. 409-417.

CoppINS B.J. Rinodina griseosoralifera, a new corticolous sorediate lichen from Western Europe // Lichenologist. - 1989. - Vol. 21. - P. 217-227.

CoppIns B.J., James P. New or interesting British lichens III // Lichenologist. - 1979. - Vol. 11. - P. $27-45$.

COPPINS B.J., JAMES P. New or interesting British lichens V // Lichenologist. - 1984. - Vol. 16. - P. 241-264.

COPPINS B.J., JAMES P.W., HAWKSWORTH D.L. New species and combinations in "The lichen flora of Great Britain and Ireland" // Lichenologist. - 1992. - Vol. 24. - P. 351-369.

Coppins B.J., Kondratyuk S. Ya., Khodosovtsev A. Ye., Zelenko S. D., Coppins A. M., Wolseley P.A. VIRCHENKO V.M. Diversity of Lichens and Bryophytes in Regional Landscape Park 'Stuzhytzia' (Ukrainian part of the International Biosphere Reserve 'Eastern Carpathians') // Lobarion lichens as indicators of the primeval forests of the Eastern Carpathians. - Darvin Intern. Workshop, 25-30 May, 1998, Kostrino, Ukraine. - K.: Phytosociocentre, 1998. - P. 139-161.

Coppins B., Kondratyuk S.YA., Khodosovtsev A.Ye, Wolseley P., Zelenko S.D. New for Crimea and Ukraine Species of the lichens // Укр. ботан. журн. - 2001. - Т. 58, № 6. - С. 716-722.

Degelius G. The Lichen Flora of the island of Anholt, Denmark // Acta Reg. Soc. Sc. Litt. Gothoburg., Bot. 1992. - N. 2. - P. 1-127.

DiEDERICH P., SCHEIDEGGER CH. Reichlingia leopoldii gen. nov. et sp. nov. a new lichenicolous hyphomycete from Central Europe // Bull. Soc. Nat. Luxemb. - 1996. - N 97. - P. 3-8.

JAMES P. New or interesting British lichens I. // Lichenologist. - 1971. - Vol. 5. - P. 114-148.

JøRGENSEN P.M., JAMES P. Studies on same Leptogium species of western Europe // Lichenologist. - 1983. Vol.15. - P.109-125.

KONDRATYUK S. YA. \& COPPINS B.J. Lichens in the Ukrainian part of the interantional biosphere reserve "Eastern Carpathians": history of the study and problems of their protection //Roczniki Bieszczadskie, 1998 (1999a), 7: 111-119.

KONDRATYUK S. YA. \& COPPINS B.J. Active protection of the endangered Lobaria pulmonaria in the Ukrainian part of the International biosphere reserve "Eastern Carpathians"//Roczniki Bieszczadskie, 1998 (1999b), 7: 349-358.

Kondratyuk S. YA., Coppins B.J., Zelenko S. D., Khodosovtsev A. Ye., Coppins A. M., Wolseley P.A. Lobarion lichens as indicators of primeval forests in the Ukrainian part of the proposed trilateral reserve "Eastern Carpathians" // Lobarion lichens as indicators of the primeval forests of the Eastern Carpathians. - Darvin Intern. Workshop, 25-30 May, 1998, Kostrino, Ukraine. - K.: Phytosociocentre, 1998. - P. 64-79.

KONDRATYUK S., COPPINS B. Basement for the lichen monitoring in Uzhansky National Nature Park, Ukrainian part of he Biosphere Reserve "Eastern Carpathians" // Roczniki Bieszczadskie. -1999 (2000). - Vol. 8. - P. 149-192.

Kondratyuk S.Ya., Popova L.P., LACKOvicova A. \& PišÚt I. 2003: A Catalogue of the Eastern Carpathian Lichens. - Kiev-Bratislava: M.H. Kholodny Institute of Botany, 2003. - 264 pp.

LAUNDON J. R. The species of Leproloma // The name for the Lepraria membranacea group // Lichenologist. 1989. - Vol. 21. - P. 1-22.

LAUNDON J. R. Lepraria in the British Isles // Lichenologist. - 1992. - Vol. 24. - P. 315-350.

LUMBSCH H.T. Die holarktischen Vertreter der Flechtengattung Diploschistes (Thelotremataceae) // J. Hattori Bot. Lab. - 1989. - Vol. 66. - P. 133-196.

LÜCKING R., SÉRUSIAUX E., VĚZDA A. Phylogeny and systematics of the lichen family Gomphillaceae (Ostropales) inferred from cladistic analysis of phenotype data // Lichenologist. - 2005. - Vol. 37, N 2. - P. 123-170.

Moberg R. The lichen genus Physcia and allied genera in Fennoscandia // Symb. Bot. Upsal. - 1977. - Vol. 22 , N 1. - P. 1-108.

NiMIS P.L. The lichens of Italy. An annotated catalogue. - Monografie XII. - Torino, 1993. - 897 p.

NovaK J. Porosty (Lichens). Physciaceae s. str. // Flora Polsca. - Vol. 6, N 3. - 1994. - 128 s.

PRINTZEN C. Die Flechtengattung Biatora in Europe // Bibl. Lichenol. - 1995. - Vol. 60. - P. 1-275.

Purvis O.W., Coppins B.J., Hawksworth D.L., James P.W., Moore D.M. The lichen flora of Great Britain and Irland // Nat. Hist. Mus. Publ. - London, 1992. - 710 p.

SÉrusiauX E., Coppins B.J., Diederich P., SCHEIDEGGer C. Fellhanera gyrophorica, a new European species with conspicuous pycnidia //Lichenologist. - 2001. - Vol. 33. - P.285-289.

Stoiko S., TASenkevich L., Shushnyak V., KrycheVska D. Regional landscape park “Stuzhytzia' - the Ukrainian part of the international biosphere reserve "Eastern Carpathians" (ecological characteristic) // Lobarion lichens as indicators of the primeval forests of the Eastern Carpathians. - Darvin Intern. Workshop, 25-30 May, 1998, Kostrino, Ukraine. - K.: Phytosociocentre, 1998. - P. 122-135.

SwInscow T. D. V. Pyrenocarpous lichens: The genus Porina in the British Isles // Lichenologist. - 1962. - Vol. 2. - P. 6-56. 
TEHLER A. Additional notes to the lichen genus Schismatomma (Arthoniales) // Mycotaxon. - 1994. - Vol. 51. P. 31-34.

TiBell L. The genus Microcalicium // Botaniska Notiser. - 1978. - Vol. 131. - P. 229-246.

TIMDAL E. The genus Hypocenomyce (Lecanorales, Lecideaceae) with special emphasis on the Norwegian and Swedisch species // Nordic J. Bot. - 1984 a. - Vol. 4. - P. 83-108.

Torrente P., EgeA J.M. La Familia Opegraphaceae en el Area Mediterranea de la Peninsula Iberica y Norte de Africa // Bibliotheca Lichenologica. - 1989. - Vol. 32. - P. 1-282.

TøNSBERG T. The sorediate and isidiate, corticolous, crustose lichens in Norway // Sommerfeltia. - 1992. - Vol. 14. - P. 1-331.

Tønsberg T., Holien H. Cladonia (Sect. Cocciferae) norvegica, a new lichen species // Nordic Journal of Botany. - 1984. - Vol. 4. - P. 79-82.

Tschermak-Woess E., Poelt J. Vezdaea, a peculiar lichen genus, and its phycobiont. // In: B.H. Brown, D.L. Hawksworth \& R.H. Bailey (eds.) Lichenology: Progress and Problems. - Academia Press, London, 1976. - P. 89-105.

VĚZDA A. Neue Gattungen der Familie Lecideaceae s. lat. (Lichenes) // Folia geobot. phytotax. Bohemoslavica. - 1986. - Vol. 22. - P. 71-83.

VĚZDA A. Lichenes Rariores Exsiccati Fasciculus sextus (numeris 51-60). - Brno, 1993. - 4 p.

VĚZDA, A., PIŠUt, I., Zwei neue Arten der Flechtengattung Absconditella (lichenisierte Stictidaceae, Ostropales) in der Tschechoslowakei // Nova Hedwigia. - 1984. - Vol. 40. - P. 341-346.

Рекомендує до друку

М.Ф. Бойко

Author's address:

Coppins B.J.

Royal Botanic Garden Edinburgh, 20 A Inverleith

Row, Edinburg EH3 5 RL, Scotland, UK

e-mail:B.Coppins@rbge.org.uk

lichensEL@btopenworld.com

Kondratyuk S.Ya.

M.H. Kholodny Institute of Botany, Tereshchenkivska

str. 2 ,

01601 Kiev-MSP-1,

Ukraine

e-mail:ksya_net@ukr.net

Khodosovtsev A.Ye.

Kherson State University

27, 40 Let Oktyabrya str.

73000, Kherson

Ukraine

e-mail: khodosovtsev@ksu.ks.ua

Zelenko S.D.

M.H. Kholodny Institute of Botany, Tereshchenkivska

str. 2,

01601 Kiev-MSP-1,

Ukraine

Wolseley P.A.

The natural History Museum, Cromwell Road,

London SW7 5 BD,

England, UK

e-mail:P.Wolseley@nhm.ac.uk
Отримано 15.11.2005 p.

Aдреси авторів:

Коппінс Б.Дж.

Королівський ботанічний сад Единбурга, 20 a

Інверлейт Роу, Единбург ЕH 35 RL,

Шотландія, $C K$

e-mail: B.Coppins@rbge.org.uk

lichensEL@btopenworld.com

Кондратюк С.Я.

Інститут ботаніки ім. М.Г. Холодного

вул. Терещенківська, 2

01601, Киї-МСП-1,

Украӥна

e-mail: ksyanet@ukr.net

Ходосовиев О. $С$.

Херсонський державний університет

вул. 40 років Жовтня, 27

73000, Херсон

Україна

e-mail:khodosovtsev@ksu.ks.ua

Зеленко С.Д.

Інститут ботаніки ім.. М.Г. Холодного

вул. Терещенківська, 2

016016 Київ-МСП-1,

Україна

Уолсели П.А.

Природничо-історичний музей

Кромвел Роад, Лондон, SW 5 BD,

Англія, СК

e-mail:P.Wolseley@nhm.ac.uk 